NEIP-99-002

hep-th/9902107

February 1999

\title{
An Effective Supergravity for the Thermal Phases of $\mathrm{N}=4$ Strings
}

\author{
J.-P. Derendinger ${ }^{\star}$ \\ Institut de Physique \\ Université de Neuchâtel \\ CH-2000 Neuchâtel, Switzerland
}

\begin{abstract}
A universal effective supergravity Lagrangian describing the thermal phases of heterotic strings on $T^{4} \times S^{1}$, IIA and IIB strings on $K^{3} \times S^{1}$ is constructed. The resulting non-perturbative phase structure is discussed.
\end{abstract}

To appear in the Proceedings of the 6th Hellenic School and Workshop on Elementary Particle Physics, Corfu, Greece, September 1998.

\footnotetext{
* Research supported in part by the European Union under the TMR contract ERBFMRX-CT96-0045 and by the Swiss Office for Education and Science.
} 


\section{Introduction}

String theories develop instabilities at high temperature $T$. The $T$-dependent mass of some winding states becomes tachyonic at the Hagedorn temperature. This phenomenon has been described [1, 2, 3] in perturbative string theories by identifying the (modular invariant) modification at finite temperature of the string partition function. The mechanism is similar to a $S^{1}$ compactification of (euclidean) time with radius $R=(2 \pi T)^{-1}$, supplemented by a spin-dependent periodicity phase related, because of modular invariance, to a modification of the GSO projections. Supersymmetry spontaneously breaks by a stringy Scherk-Schwarz mechanism.

In five dimensions, heterotic strings on $T^{4} \times S^{1}$, IIA and IIB strings on $K_{3} \times S^{1}$ are related by $S$ - and $T$-dualities. An extension of the perturbative description of strings at finite temperature with a universal (duality-invariant) temperature modulus should then display an interesting non-perturbative structure of thermal phases. This contribution reviews some aspects of this analysis, as performed in ref. [4] (where a more complete list of references can be found). Other aspects are described in the contribution of Costas Kounnas [5] to these proceedings.

The starting point of the construction is the BPS mass formula for $N_{4}=4$ fourdimensional strings, and its temperature deformation. In terms of the (heterotic) string coupling $g_{H}$ and the $T^{2}$ torus radii $R$ and $R_{6}$, the supersymmetric mass formula is [6]:

$$
\begin{aligned}
\mathcal{M}^{2} & =\left[\frac{m}{R}+\frac{n R}{\alpha_{H}^{\prime}}+g_{H}^{-2}\left(\frac{\tilde{m}^{\prime}}{R_{6}}+\frac{\tilde{n}^{\prime} R_{6}}{\alpha_{H}^{\prime}}\right)\right]^{2}+\left[\frac{m^{\prime}}{R_{6}}+\frac{n^{\prime} R_{6}}{\alpha_{H}^{\prime}}+g_{H}^{-2}\left(\frac{\tilde{m}}{R}+\frac{\tilde{n} R}{\alpha_{H}^{\prime}}\right)\right]^{2} \\
& =\frac{\left|m+n t u+i\left(m^{\prime} u+n^{\prime} t\right)+i s\left[\tilde{m}+\tilde{n} t u-i\left(\tilde{m}^{\prime} u+\tilde{n}^{\prime} t\right)\right]\right|^{2}}{\alpha_{H}^{\prime} t u} .
\end{aligned}
$$

The integers $m, n, m^{\prime}, n^{\prime}$ are the four electric momentum and winding numbers for the four $U(1)$ charges from $T^{2}$ compactification. The numbers $\tilde{m}, \tilde{n}, \tilde{m}^{\prime}, \tilde{n}^{\prime}$ are their magnetic non-perturbative partners, from the heterotic point of view. In the finite temperature case, the radius $R$ is identified with the inverse temperature, $R=(2 \pi T)^{-1}$. The mass formula reads then:

$$
\mathcal{M}_{T}^{2}=\left(\frac{m+Q^{\prime}+\frac{k p}{2}}{R}+k T_{p, q, r} R\right)^{2}-2 T_{p, q, r} \delta_{|k|, 1} \delta_{Q^{\prime}, 0}
$$

where we have set $m^{\prime}=n^{\prime}=\tilde{m}=\tilde{n}=0$ to retain only the lightest states and $Q^{\prime}$ is the (space-time) helicity charge. The integer $k$ is the common divisor of $\left(n, \tilde{m}^{\prime}, \tilde{n}^{\prime}\right) \equiv$ $k(p, q, r)$ and $T_{p, q, r}$ is an effective string tension

$$
T_{p, q, r}=\frac{p}{\alpha_{H}^{\prime}}+\frac{q}{\lambda_{H}^{2} \alpha_{H}^{\prime}}+\frac{r R_{6}^{2}}{\lambda_{H}^{2}\left(\alpha_{H}^{\prime}\right)^{2}},
$$

with $\lambda_{H}^{2}=g_{H}^{2} R R_{6} / \alpha_{H}^{\prime}$ (six-dimensional heterotic string coupling). Note that $\tilde{m}^{\prime}=k q$ corresponds to the wrapping number of the heterotic five-brane around $T^{4} \times S_{R}^{1}$, while $\tilde{n}^{\prime}=k r$ corresponds to the same wrapping number after performing a T-duality along the $S_{R_{6}}^{1}$ direction, which is orthogonal to the five-brane. All winding numbers $n, \tilde{m}^{\prime}, \tilde{n}^{\prime}$ 
correspond to magnetic charges from the field theory point of view. Their masses are proportional to the temperature radius $R$ and are not thermally shifted.

A nicer expression of the effective string tension $T_{p, q, r}$ is:

$$
T_{p, q, r}=\frac{p}{\alpha_{H}^{\prime}}+\frac{q}{\alpha_{I I A}^{\prime}}+\frac{r}{\alpha_{I I B}^{\prime}}
$$

where $\alpha_{H}^{\prime}=2 \kappa^{2} s, \alpha_{I I A}^{\prime}=2 \kappa^{2} t$ and $\alpha_{I I B}^{\prime}=2 \kappa^{2} u$ when expressed in Planck units.

The mass formula (2) possesses the same duality properties as the zero-temperature expression (国). In addition, $R$, the inverse temperature, is a duality-invariant quantity. Eq. (2) gives the states and critical values of the temperature radius at which a tachyon appears. Each corresponds to the Hagedorn transition of a perturbative string, either heterotic, or IIA or IIB. It also contains new information (on critical values of $\lambda_{H}$ and/or $R_{6}$ ) since it also decides which tachyon arises first when $T \sim 1 / R$ increases.

\section{Four-dimensional effective supergravity}

Five-dimensional strings at finite temperature can effectively be described by a fourdimensional supergravity, in which supersymmetry is spontaneously broken by thermal effects. Since we want to limit ourselves to the description of instabilities, it is sufficient to only retain, in the full $N_{4}=4$ spectrum, the potentially massless and tachyonic states, as given by mass formula (2). We can then consider only spin 0 and 1/2 states in chiral multiplets coupled to $N_{4}=1$ supergravity円.

The scalar manifold of a generic, unbroken, $N_{4}=4$ theory is [7]- $[10$

$$
\left(\frac{S l(2, R)}{U(1)}\right)_{S} \times G / H, \quad G / H=\left(\frac{S O(6, r+n)}{S O(6) \times S O(r+n)}\right)_{T_{I}, \phi_{A}} .
$$

The manifold $G / H$ of the vector multiplets splits into a part that includes the $6 r$ moduli $T_{I}$, and a second part with the infinite number $n \rightarrow \infty$ of BPS states $\phi_{A}$.

In the manifold $G / H$, we are only interested in keeping the six BPS states $Z_{A}^{ \pm}$, $A=1,2,3$, which generate thermal instabilities in heterotic, IIA and IIB strings. For consistency ${ }^{2}$, these states must be supplemented by two moduli $T$ and $U$ among the $T_{I}$ 's. We consider heterotic and type II strings respectively on $T^{4} \times S_{6}^{1} \times S_{5}^{1}$ and $K_{3} \times S_{6}^{1} \times S_{5}^{1}$, where $S_{6}^{1}$ is a trivial circle and $S_{5}^{1}$ is the temperature circle. The moduli $T$ and $U$ describe the $T^{2} \equiv S_{5}^{1} \times S_{6}^{2}$ torus. Thus, $r+n=8$ in the $N_{4}=4$ manifold (4). To construct the appropriate truncation of the scalar manifold $G / H$, we use a $Z_{2} \times Z_{2}$ subgroup contained in the $S O(6)$ R-symmetry of the coset $G / H$ for projecting out non-invariant states of the $N_{4}=4$ theory with $r+n=8$.

A single $Z_{2}$ would split $H=S O(6) \times S O(8)$ in $[S O(2) \times S O(2)] \times[S O(4) \times S O(6)]$, and the scalar manifold would become

$$
\left(\frac{S l(2, R)}{U(1)}\right)_{S} \times\left(\frac{S l(2, R)}{U(1)}\right)_{T} \times\left(\frac{S l(2, R)}{U(1)}\right)_{U} \times\left(\frac{S O(4,6)}{S O(4) \times S O(6)}\right)_{\phi_{A}} .
$$

\footnotetext{
${ }^{1}$ The four gravitinos remain degenerate at finite temperature.

${ }^{2}$ See formula (2) which depends on three moduli $s=\operatorname{Re} S, t=\operatorname{Re} T$ and $u=\operatorname{Re} U$.
} 
At this stage, the theory would have $N_{4}=2$ supersymmetry and the first three factors in the scalar manifold are vector multiplet couplings with prepotential $\mathcal{F}=i S T U / X_{0}$. The last one is a quaternionic coupling of hypermultiplets. The second $Z_{2}$ projection acts on this factor and reduces it to

$$
\left(\frac{S O(2,3)}{S O(2) \times S O(3)}\right)_{Z_{A}^{+}} \times\left(\frac{S O(2,3)}{S O(2) \times S O(3)}\right)_{Z_{A}^{-}}, \quad A=1,2,3 .
$$

This is a Kähler manifold for chiral multiplets coupled to $N_{4}=1$ supergravity. The second $Z_{2}$ projection also truncates $N_{4}=2$ vector multiplets into $N_{4}=1$ chiral multiplets.

The structure of the truncated scalar manifold and the Poincaré $N_{4}=4$ constraints on the scalar fields [9] indicate that the Kähler potential can be written as [2, 11]

$$
K=-\log \left(S+S^{*}\right)-\log \left(T+T^{*}\right)-\log \left(U+U^{*}\right)-\log Y\left(Z_{A}^{+}, Z_{A}^{+*}\right)-\log Y\left(Z_{A}^{-}, Z_{A}^{-*}\right),
$$

with

$$
Y\left(Z_{A}^{ \pm}, Z_{A}^{ \pm *}\right)=1-2 Z_{A}^{ \pm} Z_{A}^{ \pm *}+\left(Z_{A}^{ \pm} Z_{A}^{ \pm}\right)\left(Z_{B}^{ \pm *} Z_{B}^{ \pm *}\right)
$$

This Kähler function can be determined for instance by comparing the gravitino mass terms in the $N_{4}=1$ Lagrangian and inthe $Z_{2} \times Z_{2}$ truncation of $N_{4}=4$ supergravity.

The $N_{4}=1$ effective supergravity is defined by $K$ and a holomorphic superpotential $W$. And $N_{4}=4$ supergravity is defined once a field representation of the scalar manifold has been found, by specifying a gauging [9] which in particular generates the scalar potential and gravitino mass terms. Finding the correct gauging for finite temperature $N_{4}=4$ strings involves some guesswork [2, 11] and its translation into the superpotential is again easily obtained by comparing gravitino mass terms. For mass formula (2), we find:

$$
W=2 \sqrt{2}\left[\frac{1}{2}\left(1-Z_{A}^{+} Z_{A}^{+}\right)\left(1-Z_{B}^{-} Z_{B}^{-}\right)+(T U-1) Z_{1}^{+} Z_{1}^{-}+S U Z_{2}^{+} Z_{2}^{-}+S T Z_{3}^{+} Z_{3}^{-}\right] .
$$

Notice that the same effective Lagrangian could have been derived at the $N_{4}=2$ level, using a single $Z_{2}$ truncation of the theory.

Eqs. (77 9) define a supergravity theory which includes in its solutions the thermal phases of five-dimensional $N_{4}=4$ heterotic, type IIA and type IIB strings. It possesses all duality properties expected from these theories. We now turn to a survey of the most interesting phases.

\section{Masses in the low-temperature phase}

The $N=1$ supergravity scalar potential generated by the Kähler function (7) and the superpotential (9) can be written

$$
V=\kappa^{-4} e^{K} \Delta, \quad \Delta=\Delta_{S}+\Delta_{T}+\Delta_{U}+\Delta_{+}+\Delta_{-} .
$$

Each contribution $\Delta_{S}, \Delta_{T}, \ldots$, to the potential is a polynomial in the fields $S, T, U$ and $Z_{A}^{ \pm}$. In addition, $V$ only depends on quadratic combinations of $Z_{A}^{ \pm}$and their 
conjugates. It is then invariant under $Z_{A}^{ \pm} \rightarrow-Z_{A}^{ \pm}$and stationary at $Z_{A}^{ \pm}=0$ with respect to these fields. Since $V\left(S, T, U, Z_{A}^{ \pm}=0\right) \equiv 0$, it is also stationary for all values of $S, T$ and $U$. This vacuum will be stable as long as the masses (squared) of the fields $Z_{A}^{ \pm}$remain positive. This will be the case for low temperature, weak string couplings and intermediate values of the compactification radius $R_{6}$.

The low-temperature phase is common to the three strings, it is to some extent a self-dual phase. This is also visible in the pattern of supersymmetry breaking. Since

$$
\left(\mathcal{G}_{S}^{S}\right)^{-1 / 2} \mathcal{G}_{S}=\left(\mathcal{G}_{T}^{T}\right)^{-1 / 2} \mathcal{G}_{T}=\left(\mathcal{G}_{U}^{U}\right)^{-1 / 2} \mathcal{G}_{U}=-1
$$

(other derivatives of $\mathcal{G}=K+\log |W|^{2}$ vanish), the canonically normalized Goldstino is the sum of the fermionic partners of $S, T$ and $U$. And the gravitino mass is

$$
m_{3 / 2}^{2}=\kappa^{-2} e^{\mathcal{G}}=\frac{1}{4 \kappa^{2} s t u}=\frac{1}{4 R^{2}}=\frac{1}{2 \alpha_{H}^{\prime} t u}=\frac{1}{2 \alpha_{I I A}^{\prime} s u}=\frac{1}{2 \alpha_{I I B}^{\prime} s t} .
$$

The scalar and fermion mass matrices split into four sectors: $Z_{1}^{ \pm}$(heterotic winding modes), $Z_{2}^{ \pm}$(IIA windings), $Z_{3}^{ \pm}$(IIB windings) and $S, T, U$ (moduli). As already mentioned, the moduli sector is trivially massless since the potential at $Z_{A}^{ \pm}=0$ is flat.

In the $Z_{1}^{ \pm}$sector, comparing with the perturbative heterotic mass formula, the eigenstates and their masses can be identified with states with momentum and winding numbers $m$ and $n$ :

$$
\begin{array}{llll}
\frac{1}{2} \operatorname{Re}\left(Z_{1}^{+}+Z_{1}^{-}\right): & \operatorname{mass}^{2}=\frac{1}{2 \alpha_{H}^{\prime}}\left[t u+(t u)^{-1}-6\right], & m= \pm 1, & n=\mp 1 ; \\
\frac{1}{2} \operatorname{Im}\left(Z_{1}^{+}-Z_{1}^{-}\right): & \operatorname{mass}^{2}=\frac{1}{2 \alpha_{H}^{\prime}}\left[t u+(t u)^{-1}-2\right], & m=0, & n= \pm 1 ; \\
\frac{1}{2} \operatorname{Re}\left(Z_{1}^{+}-Z_{1}^{-}\right): & \operatorname{mass}^{2}=\frac{1}{2 \alpha_{H}^{\prime}}\left[t u+(t u)^{-1}+2\right], & m=0, & n= \pm 1 ; \\
\frac{1}{2} \operatorname{Im}\left(Z_{1}^{+}+Z_{1}^{-}\right): & \operatorname{mass}^{2}=\frac{1}{2 \alpha_{H}^{\prime}}\left[t u+9(t u)^{-1}-2\right], & m= \pm 1, & n= \pm 1 .
\end{array}
$$

The first state only can become tachyonic if $(\sqrt{2}-1)^{2} \leq t u \leq(\sqrt{2}+1)^{2}$. The existence of an upper and a lower bound with inverse critical values of $t u$ is a manifestation of heterotic temperature duality (a $T$-duality).

In the $Z_{2}^{ \pm}$sector, the eigenstates are perturbative IIA states with momentum and winding numbers $m$ and $\tilde{m}^{\prime}$ :

$$
\begin{array}{llll}
\frac{1}{2} \operatorname{Re}\left(Z_{2}^{+}+Z_{2}^{-}\right): & \operatorname{mass}^{2}=\frac{1}{2 \alpha_{I I A}^{\prime}}[s u-4], & m=0, & \tilde{m}^{\prime}= \pm 1 ; \\
\frac{1}{2} \operatorname{Im}\left(Z_{2}^{+}+Z_{2}^{-}\right): & \operatorname{mass}^{2}=\frac{1}{2 \alpha_{I I A}^{\prime}}\left[s u+4(s u)^{-1}\right], & m= \pm 1, & \tilde{m}^{\prime}= \pm 1 ; \\
\frac{1}{2} \operatorname{Im}\left(Z_{2}^{+}-Z_{2}^{-}\right): & \operatorname{mass}^{2}=\frac{1}{2 \alpha_{I I A}^{\prime}}\left[s u+4(s u)^{-1}\right], & m= \pm 1, & \tilde{m}^{\prime}= \pm 1 ; \\
\frac{1}{2} \operatorname{Re}\left(Z_{2}^{+}-Z_{2}^{-}\right): & \operatorname{mass}^{2}=\frac{1}{2 \alpha_{I I A}^{\prime}}[s u+4], & m=0, & \tilde{m}^{\prime}= \pm 1 .
\end{array}
$$

Again, the first state only is tachyonic if $s u<4$. Finally, in the $Z_{3}^{ \pm}$sector, the scalar mass matrix is obtained by replacing $u$ by $t$ in the $Z_{2}^{ \pm}$mass matrix, as a result of IIAIIB duality. The discussion of the mass spectrum is then similar for string states with momentum number $m$ and IIB winding $\tilde{n}^{\prime}$. Again, thermal instabilities are generated in the field direction $\frac{1}{2} \operatorname{Re}\left(Z_{3}^{+}+Z_{3}^{-}\right)$only, if $s t<4$. 
Using the variables

$$
\xi_{1}=t u=\frac{2 R^{2}}{\alpha_{H}^{\prime}}, \quad \xi_{2}=s u=\frac{2 R^{2}}{\alpha_{I I A}^{\prime}}, \quad \xi_{3}=s t=\frac{2 R^{2}}{\alpha_{I I B}^{\prime}},
$$

suggested by mass formula (2), the vacuum with $Z_{A}^{ \pm}=0$ is stable when

$$
T=\frac{1}{2 \pi \kappa}\left(\frac{1}{s t u}\right)^{1 / 2}<\frac{(\sqrt{2}-1)^{1 / 2}}{4 \pi \kappa} .
$$

It is then the low-temperature phase. Since the (four-dimensional) heterotic, IIA and IIB couplings are respectively

$$
s=\sqrt{2} g_{H}^{-2}, \quad t=\sqrt{2} g_{A}^{-2}, \quad u=\sqrt{2} g_{B}^{-2},
$$

this phase exists in the perturbative regime of all three strings. The relevant light thermal states are just the massless modes of the five-dimensional $N_{4}=4$ supergravity, with thermal mass scaling like $1 / R \sim T$.

\section{High-temperature heterotic phase}

This phase is defined by

$$
\xi_{H}>\xi_{1}>\frac{1}{\xi_{H}}, \quad \xi_{2}>4, \quad \xi_{3}>4,
$$

with $\xi_{H}=(\sqrt{2}+1)^{2}$. The domain of the moduli space that avoids type II instabilities is defined by the inequalities $\xi_{2,3}>4$. In heterotic variables,

$$
2 \pi T<\frac{1}{2 \alpha_{H}^{\prime} g_{H}^{2}} \min \left(R_{6} ; \alpha_{H}^{\prime} / R_{6}\right)=\frac{1}{4 \sqrt{2} \kappa^{2}} \min \left(R_{6} ; \alpha_{H}^{\prime} / R_{6}\right) .
$$

Type II instabilities are unavoidable when $T>T_{\text {self-dual }}$, with

$$
2 \pi T_{\text {self-dual }}=\frac{1}{2 g_{H}^{2} \sqrt{\alpha_{H}^{\prime}}}=\frac{2^{1 / 4}}{4 \kappa g_{H}} .
$$

The high-temperature heterotic phase cannot be reached for any value of the radius $R_{6}$ if the (lowest) heterotic Hagedorn temperature is higher than $T_{\text {self-dual, which translates }}$ into

$$
g_{H}^{2}>g_{\text {crit. }}^{2}=\frac{\sqrt{2}+1}{2 \sqrt{2}} \sim 0.8536 .
$$

Only type II thermal instabilities exist in this strong-coupling regime and the value of $R_{6} / \sqrt{\alpha_{H}^{\prime}}$ decides whether the type IIA or IIB instability will have the lowest critical

\footnotetext{
${ }^{3}$ From low temperature.
} 
temperature. If on the other hand the heterotic string is weakly coupled, $g_{H}<g_{\text {crit. }}$, the high-temperature heterotic phase is reached for values of the radius $R_{6}$ verifying

$$
2 \sqrt{2} g_{H}^{2}(\sqrt{2}-1)<\frac{R_{6}}{\sqrt{\alpha_{H}^{\prime}}}<\frac{1}{2 \sqrt{2} g_{H}^{2}(\sqrt{2}-1)} .
$$

The large and small $R_{6}$ limits, with fixed coupling $g_{H}$, again lead to either type IIA or type IIB instability.

In the region of the moduli space defined by Eqs. (15) and after minimization with respect to $Z_{A}^{ \pm}$, the potential becomes

$$
\kappa^{4} V=-\frac{1}{s} \frac{\left(\xi_{1}+\xi_{1}^{-1}-6\right)^{2}}{16\left(\xi_{1}+\xi_{1}^{-1}\right)} .
$$

It has a stable minimum for fixed $s$ (for fixed $\alpha_{H}^{\prime}$ ) at the minimum of $\xi_{1}+\xi_{1}^{-1}$ :

$$
\xi_{1}=1, \quad \kappa^{4} V=-\frac{1}{2 s} .
$$

In units of $\alpha_{H}^{\prime}$, the temperature is fixed: $\xi_{1}=1=2 R^{2} / \alpha_{H}^{\prime}=R^{2} /\left(\kappa^{2} s\right)$. The transition from the low-temperature vacuum is due to a condensation of the heterotic thermal winding mode $\operatorname{Re}\left(Z_{1}^{+}+Z_{1}^{-}\right)$, or equivalently by a condensation of type IIA NS five-brane in the type IIA picture.

At the level of the potential only, this phase exhibits a runaway behaviour in $s$. The effective supergravity is solved by a background with a dilaton linear in a space-like directionf. Its content in terms of non-critical strings is described in detail in ref. [4] ๒. We will here only consider its mass pattern and the breakdown of supersymmetry.

The high-temperature heterotic phase only exists in the perturbative domain of the heterotic string, where $s$ is the dilaton, and, by duality, in non-perturbative type II regimes. Accordingly, supersymmetry breaking arises from $s$ : only $\mathcal{G}_{S}$ is non-zero. The mass spectrum naturally splits in two sectors, the heterotic dilaton multiplet and secondly all other chiral multiplets $\left(y_{a}, \chi_{a}\right)$ that play a passive role in supersymmetry breaking.

- Fermions $\chi_{a}$ :

Since the Kähler metric is diagonal, $\mathcal{G}_{b}=0$ and $\mathcal{G}_{c b}^{S}=0$, the mass matrix simplifies to

$$
\left(\mathcal{M}_{1 / 2}\right)_{a b}=\kappa^{-1} e^{\mathcal{G} / 2}\left(\mathcal{G}^{-1 / 2}\right)_{a}^{c} \mathcal{G}_{c d}\left(\mathcal{G}^{-1 / 2}\right)_{b}^{d}=m_{3 / 2}\left(\mathcal{G}^{-1 / 2}\right)_{a}^{c} \mathcal{G}_{c d}\left(\mathcal{G}^{-1 / 2}\right)_{b}^{d}
$$

Mixings can only arise from non-zero values of $\mathcal{G}_{a b}$ due to superpotential contributions. Since $W$ includes a term proportional to $T U Z_{1}^{+} Z_{1}^{-}$, these four fields, which are nonzero at the vacuum, get mixed. Masses are completely determined (in $m_{3 / 2}$ units) since all parameters are fixed in this sector. On the other hand, fermion masses in the $Z_{2}^{ \pm}$ sector are $m_{3 / 2}[s u \pm 1]$ and $m_{3 / 2}[s t \pm 1]$ in the $Z_{3}^{ \pm}$sector.

\footnotetext{
${ }^{4}$ Type II instabilities, for $\xi_{2,3}<4$, do not stabilize the moduli $s, t$ and $u$. Finding a background for these phases left aside here is a more complicated task.

${ }^{5}$ See also ref. $[5]$.
} 
- Scalars $y_{a}$ :

Scalar masses arise from Lagrangian mass terms and also from a universal mass shift due to the linear dilaton background. The physical scalar mass matrix reads:

$$
\mathcal{M}_{0}^{2}=m_{3 / 2}^{2}\left(\left[\mathcal{G}^{-1 / 2}\right]_{e}^{a}\left[\mathcal{G}^{-1 / 2}\right]_{c}^{f}\right)\left(\begin{array}{cc}
\mathcal{G}^{e n} \mathcal{G}^{-{ }^{r}}{ }_{n} \mathcal{G}_{r g} & -2 s W^{-1} W^{e h S} \\
-2 s W^{-1} W_{f g S} & \mathcal{G}_{f m} \mathcal{G}_{p}^{-1}{ }_{p}^{m} \mathcal{G}^{p h}
\end{array}\right)\left(\begin{array}{c}
{\left[\mathcal{G}^{-1 / 2}\right]_{b}^{g}} \\
{\left[\mathcal{G}^{-1 / 2}\right]_{h}^{d}}
\end{array}\right) .
$$

Comparing with the fermion mass matrix (20), one observes that the spectrum would be supersymmetric [ without the off-diagonal term proportional to $2 s W^{-1} W^{S i j}$. Since supersymmetry breaks in the $S$ direction, these contributions generate O'Raifeartaightype bosonic mass shifts for states that couple in the superpotential to $S$ : these are the heterotic dyonic states $Z_{2}^{ \pm}$and $Z_{3}^{ \pm}$, which generate type II instabilities. Heterotic perturbative states have a supersymmmetric spectrum [2]. The supersymmetry-breaking contributions to the scalar mass spectrum imply the existence of non-perturbative modes lighter than their fermionic partners. Explicitly, mass eigenvalues in the $Z_{2}$ sector are

$$
m_{3 / 2}^{2}\left[(s u-1)^{2} \pm 2 s u\right], \quad \quad m_{3 / 2}^{2}\left[(s u+1)^{2} \pm 2 s u\right],
$$

to be compared with the fermion masses $|s u-1| m_{3 / 2}$ and $|s u+1| m_{3 / 2}$. The mass pattern in the $Z_{3}^{ \pm}$sector is obtained by substituting $u$ for $t$ in the $Z_{2}^{ \pm}$sector.

To summarize, the spectrum is supersymmetric in the perturbative heterotic and moduli sector $\left(T, U, Z_{1}^{ \pm}\right)$, and with O'Raifeartaigh pattern in the non-perturbative sectors:

$$
\begin{aligned}
& Z_{2}^{ \pm}: m_{\text {bosons }}^{2}=m_{\text {fermions }}^{2} \pm 2 s u m_{3 / 2}^{2}, \\
& Z_{3}^{ \pm}: m_{\text {bosons }}^{2}=m_{\text {fermions }}^{2} \pm 2 s t m_{3 / 2}^{2} .
\end{aligned}
$$

This phenomenon persists in the five-dimensional type IIA [and type IIB] limit, in which the $Z_{3}^{ \pm}\left[Z_{2}^{ \pm}\right]$states become superheavy and decouple while the $Z_{2}^{ \pm}\left[Z_{3}^{ \pm}\right]$ scalar masses are shifted by a non-perturbative amount, since in this limit $s u=1 / \lambda_{H}^{2}$ $\left[s t=1 / \lambda_{H}^{2}\right]$. Thus, supersymmetry appears broken by non-perturbative effects. Note, however, that this statement may not hold in the case of the four-dimensional background solution with a dilaton motion in one direction. This case has only an effective three-dimensional Poincaré invariance, which does not imply in general mass degeneracyin massive multiplets, even if local supersymmetry is unbroken [12, [13].

\section{Acknowledgements}

This report is based on work done in collaboration with I. Antoniadis and C. Kounnas. I wish to thank the organizers of the "6th Hellenic School and Workshops on Elementary Particle Physics", the European Union (contracts TMR-ERBFMRX-CT96-0045 and -0090) and the Swiss Office for Education and Science for financial support.

\footnotetext{
${ }^{6}$ In the sense of equal boson and fermion masses.
} 


\section{References}

[1] B. Sathiapalan, Phys. Rev. D35 (1987) 3277;

Ya. Kogan, JETP Lett. $4 \underline{5}$ (1987) 709;

J. J. Atick and E. Witten, Nucl. Phys. B310 (1988) 291;

C. Kounnas and B. Rostand, Nucl. Phys. B341 (1990) 641.

[2] I. Antoniadis and C. Kounnas, Phys. Lett. B261 (1991) 369.

[3] E. Alvarez, Phys. Rev. D31 (1985) 418;

B. Sundborg, Nucl. Phys. B254 (1985) 583;

M. J. Bowick and L. C. R. Wijewardhana, Phys. Rev. Lett. $\underline{54}$ (1985) 2485;

S. H.-H. Tye, Phys. Lett. B158 (1985) 388;

I. Antoniadis, J. Ellis and D. V. Nanopoulos, Phys. Lett. B199 (1987) 402;

M. Axenides, S. D. Ellis and C. Kounnas, Phys. Rev. D37 (1988) 2964.

[4] I. Antoniadis, J.-P. Derendinger and C. Kounnas, hep-th/9902032, submitted to Nucl. Phys. B.

[5] C. Kounnas, these proceedings, hep-th/9902072.

[6] J. H. Schwarz and A. Sen, Phys. Lett. B312 (1993) 105; Nucl. Phys. B411 (1994) 35 ;

A. Sen, Phys. Lett. B303 (1993) 22; B329 (1994) 217; Nucl. Phys. B329 (1994) 217 ;

M. J. Duff, J. T. Liu and J. Rahmfeld, Nucl. Phys. B459 (1996) 125;

M. Cvetic and D. Youm, Phys. Rev. D53 (1996) R584; Phys. Lett. B359 (1995) 87 ;

K.-L. Chan and M. Cvetic, Phys. Lett. B375 (1996) 98;

M. Cvetic and A. Tseytlin, Phys. Lett. B366 (1996) 95; Phys. Rev. D53 (1996) 5619 ;

G. L. Cardoso, G. Curio, D. Lüst, T. Mohaupt and S.-J. Rey, Nucl. Phys. B464 (1996) 18;

R. Dijkgraaf, E. Verlinde and H. Verlinde, Nucl. Phys. B484 (1997) 543; Nucl. Phys. B486 (1997) 77; Nucl. Phys. B486 (1997) 89;

C. Kounnas, Nucl. Phys. B58 [Proc. Suppl.] (1997) 57;

E. Kiritsis and C. Kounnas, Nucl. Phys. B503 (1997) 117.

[7] J.-P. Derendinger and S. Ferrara, in: Supersymmetry and Supergravity '84, ed. B. de Wit, P. Fayet and P. van Nieuwenhuizen (World Scientific, Singapore, 1984), p. 159.

[8] A. H. Chamseddine, Nucl. Phys. B185 (1981) 403.

[9] M. de Roo, Nucl. Phys. B255 (1985) 515; Phys. Lett. B156 (1985) 331;

E. Bergshoeff, I.G. Koh and E. Sezgin, Phys. Lett. B155 (1985) 71.

[10] S. Ferrara and C. Kounnas, Nucl. Phys. B328 (1989) 406. 
[11] C. Kounnas and M. Porrati, Nucl. Phys. B310 (1988) 355;

S. Ferrara, C. Kounnas, M. Porrati and F. Zwirner, Nucl. Phys. B318 (1989) 75;

M. Porrati and F. Zwirner, Nucl. Phys. B326 (1989) 162;

A. Giveon and M. Porrati, Phys. Lett. B246 (1990) 54; Nucl. Phys. B3555 (1991) 422 .

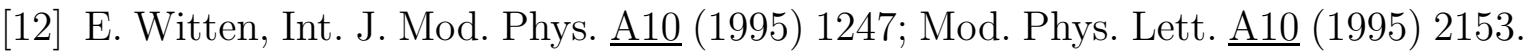

[13] K. Becker, M. Becker and A. Strominger, Phys. Rev. D51 (1995) 6603. 